OPEN ACCESS

Edited by:

Jean-Christophe Paillart,

Université de Strasbourg, France

Reviewed by:

Cristina Romero-López,

Institute of Parasitology

and Biomedicine "López-Neyra"

(CSIC), Spain

Xiao Heng,

University of Missouri, United States

*Correspondence:

Tetsuro Suzuk

tesuzuki@hama-med.ac.jp

Specialty section:

This article was submitted to

Virology,

a section of the journal

Frontiers in Microbiology

Received: 09 January 2018

Accepted: 21 February 2018

Published: 07 March 2018

Citation:

Shi G and Suzuki T (2018) Molecular Basis of Encapsidation of Hepatitis C

Virus Genome.

Front. Microbiol. 9:396.

doi: 10.3389/fmicb.2018.00396

\section{Molecular Basis of Encapsidation of Hepatitis C Virus Genome}

\author{
Guoli Shi ${ }^{1}$ and Tetsuro Suzuki2*
}

${ }^{1}$ Antiviral Immunity and Resistance Section, HIV Dynamics and Replication Program, Center for Cancer Research, National Cancer Institute, Frederick, MD, United States, ${ }^{2}$ Department of Virology and Parasitology, Hamamatsu University School of Medicine, Hamamatsu, Japan

Hepatitis C virus (HCV), a major etiologic agent of human liver diseases, is a positive-sense single-stranded RNA virus and is classified in the Flaviviridae family. Although research findings for the assembly of HCV particles are accumulating due to development of HCV cell culture system, the mechanism(s) by which the HCV genome becomes encapsidated remains largely unclear. In general, viral RNA represents only a small fraction of the RNA molecules in the cells infected with RNA viruses, but the viral genomic RNA is considered to selectively packaged into virions. It was recently demonstrated that HCV RNAs containing $3^{\prime}$ end of the genome are selectively incorporated into virus particles during the assembly process and the $3^{\prime}$ untranslated region functions as a cis-acting element for RNA packaging. Here, we discuss the molecular basis of RNA encapsidation of HCV and classical flaviviruses, contrast with the packaging mechanism of HIV-1.

Keywords: hepatitis C virus, encapsidation, packaging signal, cis-acting element, virion assembly

\section{INTRODUCTION}

Hepatitis C virus (HCV) infection is a major cause of chronic hepatitis, liver cirrhosis, and hepatocellular carcinoma worldwide. In spite of the fact that HCV is targeted by innate, cellular and humoral immune mechanisms, its long-standing persistent infection can be established in a majority of the infected individuals. The recent development of direct-acting antivirals, which target specifically $\mathrm{HCV}$ non-structural proteins, initiated the era of high efficacy and well-tolerated medications with high cure rates (Bourlière et al., 2017).

Hepatitis $\mathrm{C}$ virus is classified in the Hepacivirus genes within the Flaviviridae family, which includes the classical flaviviruses such as yellow fever virus and dengue virus. The viral particle consists of a nucleocapsid, surrounded by a lipid envelope containing two viral glycoproteins, E1 and E2 (Moriishi and Matsuura, 2016). A hallmark of HCV particles is their association with cellular lipoproteins that potentially determine both morphology and biophysical properties of the virion (Suzuki, 2017). Although significant progress has been made regarding molecular biology of HCV life cycle over the last decade, our understanding of mechanisms on virion assembly, in particular, encapsidation of the viral genome has been limited.

In this short review, we summarize our current knowledge of moleculer basis of packaging of genomic RNAs of HCV and the classical flaviviruses. We also provide an overview of the mechanism on selective packaging of the HIV-1 genome and compare it with the HCV encapsidation. 


\section{GENOME STRUCTURE OF HCV}

The genome of HCV is a positive-sense single-stranded RNA with highly structured elements, which is about $9.6 \mathrm{~kb}$ in length (Choo et al., 1989). This genomic RNA contains one single open reading frame (ORF) encoding a polyprotein which can be processed into 10 viral proteins after translation. The HCV genome was flanked by the $5^{\prime}$ untranslated- (5' UTR) and the $3^{\prime}$ untranslated( $3^{\prime}$ UTR) regions at the $5^{\prime}$ - and $3^{\prime}$ ends, respectively. Both UTRs are highly structured and crucial for the viral translation and proliferation and are well conserved among genotypes or strains of $\mathrm{HCV}$.

The $5^{\prime}$ UTR is a $~ 340$-nucleotide (nt) element composed by four highly structured domains and is implicated in almost the whole processing of HCV life cycle, except virions entry. Domain I of the $5^{\prime}$ UTR comprises a single stem-loop. Domains II to IV of the $5^{\prime}$ UTR constitute an internal ribosomal entry site (IRES), which is a prerequisite for cap-independent translation of viral RNA (Figure 1) (Wang et al., 1995; Honda et al., 1996). While in general miRNAs interact with the $3^{\prime}$ UTR of mRNAs to promote mRNA destabilization and/or translational repression, the miR-122 binding to the $5^{\prime}$ UTR of HCV RNA is essential for the viral replication (Jopling, 2008; Machlin et al., 2011). The 3' UTR varies between 200 and $235 \mathrm{nt}$ in length, including a short variable region, as well as a poly(U/UC) stretch with a length of about $90 \mathrm{nt}$, and a virtually invariant 98-nt X-tail region $\left(3^{\prime} \mathrm{X}\right)$. The $3^{\prime}$ UTR is vital for HCV genome replication. Several deletions or substitution mutations within the region resulted in loss of the viral replication (Friebe and Bartenschlager, 2002). Although the IRES of $5^{\prime}$ UTR is sufficient to initiate translation of mRNA in reporter systems, it was suggested that its translation efficiency can be elevated in the presence of $3^{\prime}$ UTR, possibly through stabilizing the RNA and forming the RNA complex with 5' UTR (Song et al., 2006; Bai et al., 2013). However, no or only a limited contribution of $3^{\prime}$ UTR to stabilization of the viral genome was observed in our trans-packaging system (Shi et al., 2016). The $3^{\prime} \mathrm{X}$ tail has been described to fold into two conformations, one of which is composed with three stem loops, while the other consists of two stem loops. In both predicted conformations, the SL I at the very end of 3 terminus is preserved, while the upstream $55 \mathrm{nt}$-long segment forms either a single stem loop exposing the dimer linkage sequence (DLS) or two stem loops (SLII and SLIII) (Figure 1) (Ivanyi-Nagy et al., 2006; Shetty et al., 2010; Palau et al., 2013; Romero-López et al., 2014). Though DLS was found inevitable to form HCV RNA homodimers in vitro, it is not yet clear whether homodimers are formed in infected cells. Since involvement of the DLS region in HCV RNA dimerization would preclude its ability to engage in the longrange kissing interaction with the NS5B-coding region that is known to be essential for RNA replication, and the HCV virion contains a single copy of the genomic RNA, DLS might be an intermediate conformation during switching between processes of genome replication and/or encapsidation. The $3^{\prime}$ tail often forms long distance complexes with RNA structures, including elements within the NS5B coding sequence in HCV genome. Among the structured RNA elements in the coding region, the cis replication element (CRE) located at NS5B region, which consists of three conserved domains, 5BSL3.1, 5BSL3.2, and 5BSL3.3, have been demonstrated for their regulatory roles in translation and genome replication via long distance interacting with $5^{\prime}$ UTR, 3' UTR and SL9033 (a structured RNA element upstream of CRE) (You et al., 2004; Friebe et al., 2005; Diviney et al., 2008; RomeroLopez and Berzal-Herranz, 2009; Shetty et al., 2010; Tuplin et al., 2012). The polyU/UC stretch provides quite a spatial flexibility to the stem-loops in $3^{\prime}$ UTR, allowing spatial contact with CRE. Thus, the length of the polyU/UC stretch as well as UTP contents in the region are important for the viral replication (You and Rice, 2008).

\section{MECHANISTIC ANALYSIS OF ENCAPSIDATION OF THE HCV GENOME}

Although evidence regarding the viral replication and particle formation of $\mathrm{HCV}$ are accumulating due to development of HCV cell culture system, detailed mechanisms for incorporation of the viral genome into progeny virions has remained largely unclear. Very few evidence on HCV RNA elements contributing to the viral genome packaging has been scattered. An early study demonstrated that the $5^{\prime}$ UTR of the genome most likely does not contain cis-acting RNA structures required for the viral encapsidation (Friebe and Bartenschlager, 2009). In our recent study, the HCV $3^{\prime}$ UTR was found to function as a cisacting element, which is important in viral genome packaging, as described below (Shi et al., 2016).

In the livers and sera of patients with $\mathrm{HCV}$ infection, in addition to $\mathrm{HCV}$ full genome RNA, a large portion of $5^{\prime}$-end subgenomic HCV RNAs were found, which were possibly generated by premature transcription termination or RNA cleavage at particular sites within the viral genome (Quadri and Negro, 2001; Shimizu et al., 2006). Accordingly, in the HCV cell culture system, we also found greater distribution of $5^{\prime}$-end subgenomic RNAs over the intact $\mathrm{HCV}$ genome. By quantitating HCV RNAs both at $5^{\prime}$ end and $3^{\prime}$ end sites, we found that the $3^{\prime} / 5^{\prime}$-end ratio of the viral RNA copies in culture supernatants of $\mathrm{HCV}$-infected cells was markedly higher than that in the infected cells, confirming over-distribution of $5^{\prime}$ end subgenomic HCV RNAs in the viral replicating cells. Further higher $3^{\prime} / 5^{\prime}$ ratios were observed in the density fractions with high infectivity rather than the whole culture supernatants, indicating a positive correlation between $3^{\prime} / 5^{\prime}$ ratio of the HCV RNA copy and the viral infectivity. Further experiments to comprehensively understand the encapsidation of HCV genome demonstrated that the size of most of RNA species extracted from purified HCV particles appears to correspond to the full-length of the viral genome by judged by the bioanalyzer electrophoresis (unpublished data). Thus, it is likely that the HCV RNA species with the genomicsize or nearly so are positively selected and incorporated into the viral particles during the encapsidation processing.

Regarding to currently revealed mechanisms of virus encapsidation, which are generally initiated via recognition of the structured element(s) within genomic nucleic acids, termed packaging signal(s), by the viral capsid or coat protein, we hypothesized that the specific packaging of $\mathrm{HCV}$ genome is 


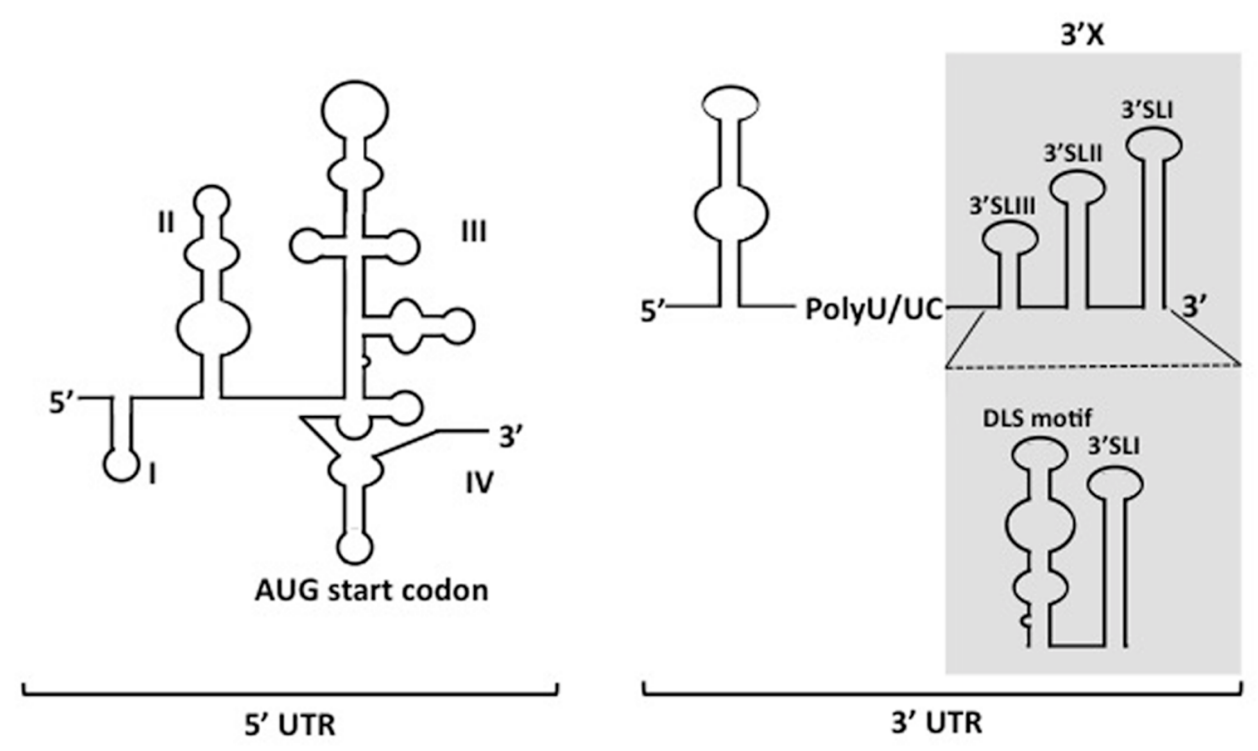

FIGURE 1 | Depicted secondary structures of $5^{\prime}$ - and $3^{\prime}$ UTRs of HCV genome. The $5^{\prime}$ UTR contains four structured domains (I-IV). The translational start codon is indicated. Two types of conformations for the $3^{\prime} X$ region (shadowed region) within the $3^{\prime}$ UTR have been proposed.

driven by interaction of the viral RNA with capsid protein, Core. Our in vitro binding assays showed that amongst the structured HCV RNA elements tested, 3' UTR possessed higher Core-binding affinity compared to other RNA elements such as $5^{\prime}$ UTR and the elements in NS5B coding region and that their affinity was independent of the lengths of RNA sequences (Shi et al., 2016). Notably, the RNA fragment with both $3^{\prime}$ UTR and CRE showed lower Core-binding affinity than that with 3' UTR alone, speculating that the long-distance kissing-loop structure consisting of CRE and stem-loop(s) within $3^{\prime}$ UTR led to masking certain motif(s) involved in the Core binding. Together with the finding that the kissing-loop interaction of the $\mathrm{HCV}$ genome is important for RNA replication, it is reasonable to consider that conformational alteration of the $3^{\prime}$-end regions of the $\mathrm{HCV}$ genome may contribute to switching the process during HCV life cycle from RNA replication to the early phase of particle formation.

Although findings from the in vitro binding assays suggested that $3^{\prime}$ UTR, rather than other RNA elements tested, is potentially implicated in the viral encapsidation, only successful directing of RNA into virions could authentically prove its role as a cis-packaging signal. By utilizing a trans-packaging system (Masaki et al., 2010) with replication-defective HCV subgenome, we found that deletion of $3^{\prime}$ UTR is deleterious for production of trans-complemented HCV particles (HCVtcp) while deletion of $5^{\prime}$ UTR also impaired it to some extent (Shi et al., 2016). RNA stability of deletion mutants used was confirmed to be comparable to that of intact subgenomes. Notably, the loop motifs of $3^{\prime} \mathrm{X}$ tails in $3^{\prime}$ UTR were found to be important for binding to Core as well as HCVtcp production. Substitutions in the loop sequences of which secondary structures were not affected, resulted in marked impairment of the Core-binding and RNA packaging into the viral particles (Shi et al., 2016).
Stewart et al. used in vitro systematic evolution of ligands by exponential enrichment (SELEX) to screen RNA aptamers that bind specifically to Core and identified 8 short RNA motifs within the HCV ORF that are possibly involved in the virion assembly or the late stages of the HCV life cycle (Stewart et al., 2016). Aptamers within a randomized library possess their unique tertiary structures, and are present in their most stable conformations. SELEX is therefore likely to enrich for aptamers with conserved conformation rather than a unique primary sequence. It is possible that multiple RNA elements are involved in genome packaging of $\mathrm{HCV}$, in addition to $3^{\prime} \mathrm{UTR}$ and $5^{\prime}$ UTR, at the physiological environment. While the motifs scattered within genome may assist the encapsidation through interacting with Core, the viral $3^{\prime}$ UTR may also play a role in recruiting cellular and/or other viral factors that are important for genome packaging or virus assembly (Harris et al., 2006). RNA aptamers selected against HCV NS3 helicase were found to share high similarities with $3^{\prime}$ UTR (Nishikawa et al., 2004). It may be possible that $3^{\prime}$ UTR undergoes conformational alteration mediated by NS3 helicase during replication events and/or at switching from replication to encapsidation. As a capsid protein, HCV Core may have an ability to facilitate the inter-conversion between diverse RNA structures, leading to regulating structural transitions of the viral RNA elements during HCV life cycle (Cristofari et al., 2004; Gawlik and Gallay, 2014). 3' UTR has been found to activate an IKK- $\alpha$-dependent pathway that induces lipogenic genes and to enhance Core-associated lipid droplet formation beneficial to the viral assembly (Li et al., 2013). Indeed, in the trans-packaging system $3^{\prime}$ UTR appeared to facilitate the interaction between Core and NS5A at or around lipid droplets. NS5A, a phosphorylated non-structural protein of $\mathrm{HCV}$, is a multi-functional protein required for RNA replication and virion assembly (Masaki and Suzuki, 2015). The Core-NS5A interaction 


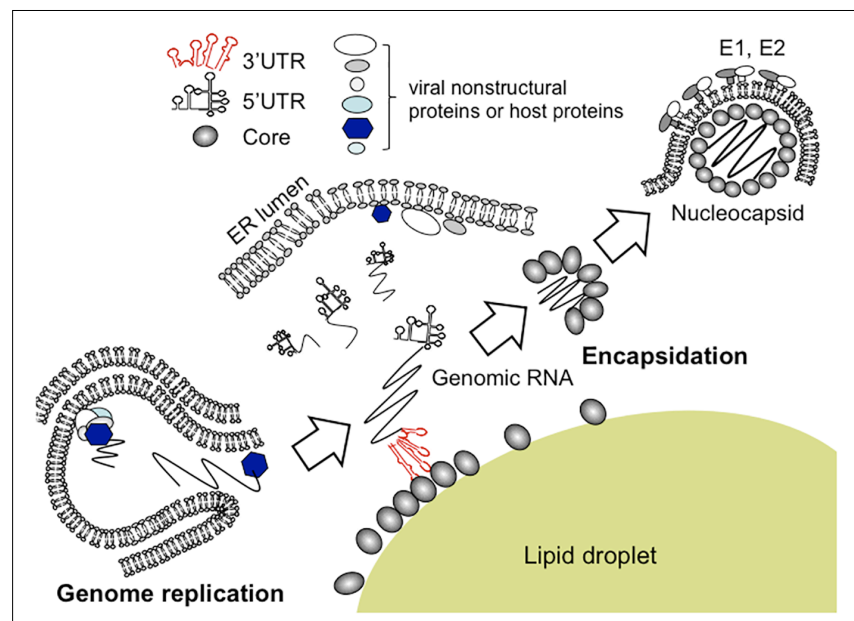

FIGURE 2 | Proposed model for encapsidation of HCV genome. Newly synthesized HCV genomic RNA is released from membrane vesicles containing the replication complex (lower left). It may be likely that the viral genome bound to NS5A is recruited to the surface of lipid droplets, enabling its interaction with Core. For nucleocapsid formation, the $3^{\prime}$ UTR of the HCV genome acts as a cis-acting element for RNA packaging. Within the $3^{\prime}$ UTR region, the loop sequences of stem-loop structures appear to be essential for HCV encapsidation.

is thought to be crucial for the early phase of the particle formation (Appel et al., 2008; Masaki et al., 2008). A proposed model of encapsidation of HCV genome is illustrated in Figure 2. between $5^{\prime}$ UTR and $3^{\prime}$ UTR of the flavivirus genome result in the cyclisation of the viral RNA, which is essential for viral replication (Alvarez et al., 2005). However, packaging signals or cis-acting elements important for RNA packaging have not been identified in the genomic RNA thus far. It has been considered that replication and encapsidation of flaviviruses are functionally coupled, which was evidenced by unable to detect infectious virus particles production with a plasmid DNA encoding replication-deficient full-length Kunji virus cDNA (Khromykh et al., 2001b). As it happens with HCV, HCVtcp production in the trans-packaging system with replicationdefective subgenome was approximately 10 -fold lower compared to that with the replication-competent setting (Shi et al., 2016). One could argue that the active replication machineries of flaviviruses and HCV not only provide continuous transcription and accumulation of genomic RNA, but also potentially recruit factors that are important for efficient encapsidation of genomic RNA. It has been described that direct interaction between $5^{\prime}$ and $3^{\prime}$ terminal nucleotide sequences of flaviviruses genome RNA mediated the virus RNA cyclization (Khromykh et al., 2001a). The significance of genome cyclization during virus life cycle has been uncovering. Evidences demonstrated that genome cyclization is implicated in regulating genome replication (Mir et al., 2006; Villordo and Gamarnik, 2009). It is still to be explored whether the cyclization is involved in flaviviruses encapsidation. This long-range RNA-RNA interaction could also serve as a molecular switch during different stage of virus replication, similarly as in HCV replication.

\section{ENLIGHTENMENT FROM STUDIES OF GENOME PACKAGING OF RETROVIRUSES}

So far, the packaging of genomic RNA of retroviruses has been better elucidated than any other RNA viruses. Herein, we will take HIV-1 as an example to briefly compare the packaging processing with that of $\mathrm{HCV}$. Upon viral entry to the target cells, the RNA genome of HIV is reverse-transcribed into doublestranded DNA, followed by integrating into the cellular genome to form a provirus (Freed, 2015). The spliced- and unspliced viral transcripts are exported from nuclear to cytoplasm for subsequent processing. Genome dimerization is vital for HIV packaging. upon dimerization, a pair of unspliced genomic RNAs were packaged into virions (Skripkin et al., 1994; Russell et al., 2003; Paillart et al., 2004). To accomplish the specific packaging of the viral genome, HIV-1 uses the cis-acting RNA elements in the viral genome and the trans-acting elements in Gag protein (D'Souza and Summers, 2005). The 5' UTR contains the packaging signal of HIV-1, psi ( $\Psi$ ) (Lever et al., 1989), which consists of a series of stem-loops, as well as a short sequence required for the initiation of RNA dimerization (McBride and Panganiban, 1996; Lu et al., 2011). A part of the 5' end of Gag-coding sequence is also involved in the encapsidation (Chamanian et al., 2013; Olson et al., 2015; Comas-Garcia et al., 2016). Packaging of HIV genome is initiated by the recognition of 
packaging signals, which is a prerequisite for assembly of HIV particles with full-length genomic RNA. It was recently unveiled that binding of Gag at the cell membrane stabilized the dimerization of two copies of the genomic RNA (Chen et al., 2016), thus paved the way for assembly particles and production of progeny viruses. The generality of the encapsidation mechanism between HIV-1 and HCV is that both use highly conserved and structured RNA elements for recognition by the viral coat protein and that, to achieve maximum packaging efficiency, multiple RNA elements within the viral genome play cooperatively while with one element as dominating packaging signal. The HIV-1 RNA packaging signal is recognized by Gag, which is synthesized as a polyprotein that is later processed into six mature proteins: matrix, capsid, SP1, nucleocapsid (NC), SP2, and p6. NC plays the leading role for the selective packaging of the HIV-1 RNA genome (Bell and Lever, 2013). Mutations of the zinc knuckle motifs in NC can cause severe defects in the viral RNA packaging (Guo et al., 2002). A recent study reported that a Gag mutant in which four basic residues between the two zinc fingers have been replaced with alanines retained significant affinity for the packaging signal, but not control RNA (Comas-Garcia et al., 2017), suggesting that these four basic residues help to maintain the proper conformation of the NC domain as well as contributing to the electrostatic interactions with RNA (Webb et al., 2013). Intriguingly, we also found that the basic residues in $\mathrm{N}$-terminus of HCV Core are critical for both specific interaction with HCV RNA and electrostatic interactions (unpublished data).

\section{FUTURE PERSPECTIVES}

There is accumulating evidence regarding nucleic acids packaged in the virus particles determined by new-generation sequencing techniques. In addition to the viral RNAs, significant amounts of host RNAs are detectable in HIV-1 particles, such as host mRNAs, tRNAs, and 7SL RNA (Telesnitsky and Wolin, 2016). Recruitment of these RNAs into HIV particles is considered to be independent of the viral genome packaging and to be mediated by the viral and/or host RNA-binding proteins through less sequence-specific interactions. In our primary analysis, host-derived RNA species were also found in HCV particles (unpublished data). How these host-cell RNAs are packaged into

\section{REFERENCES}

Alvarez, D. E., Lodeiro, M. F., Ludueña, S. J., Pietrasanta, L. I., and Gamarnik, A. V. (2005). Long-range RNA-RNA interactions circularize the dengue virus genome. J. Virol. 79, 6631-6643. doi: 10.1128/JVI.79.11.6631-6643. 2005

Annamalai, P., and Rao, A. L. (2006). Packaging of brome mosaic virus subgenomic RNA is functionally coupled to replication-dependent transcription and translation of coat protein. J. Virol. 180, 10096-10108. doi: 10.1128/JVI.011 86-06

Appel, N., Zayas, M., Miller, S., Krijnse-Locker, J., Schaller, T., Friebe, P., et al. (2008). Essential role of domain III of nonstructural protein 5A for hepatitis
$\mathrm{HCV}$ virus particles as well as the virological significance of nonviral nucleic acids present in the virus particles remains to be elucidated.

For several positive-strand RNA viruses; not only flavivirus (Khromykh et al., 2001b), but poliovirus (Nugent et al., 1999) and bromovirus (Annamalai and Rao, 2006), functionally coupling of RNA packaging and replication has been reported. It is likely that a replication-coupled packaging mechanism potentially permits efficient access of the capsid protein to interact with progeny viral RNAs. Functional active replication machinery might also recruit factors promoting encapsidation efficiently at the site for nucleocapsid formation. However, this coupling mechanism might result in competing between capsid protein and the replicase for RNA binding. To advance our understanding of the encapsidation of positive-strand RNA viruses, further researches on their regulatory mechanisms for the link between the RNA replication cycle and virus assembly are required. Specific recognition of the genomic RNA via the packaging signal and coupling with replication are not mutually exclusive, but seem rather cooperate for the best beneficial of encapsidation and nuclecapsid formation. Our methodology in investigating $\mathrm{HCV}$ encapsidation could be applied to explore the fundamental cis-acting RNA elements that are crucial for the genome packaging of flaviviruses or other positive-strand RNA viruses.

\section{AUTHOR CONTRIBUTIONS}

All authors listed have made a substantial, direct and intellectual contribution to the work, and approved it for publication.

\section{FUNDING}

This work was supported by the Japan Agency for Medical Research and Development, AMED and from the Ministry of Education, Culture, Sports, Science, and Technology, Japan.

\section{ACKNOWLEDGMENTS}

We are grateful to T. Mochizuki (Department of Virology and Parasitology, Hamamatsu University School of Medicine) for secretarial work.

C virus infectious particle assembly. PLoS Pathog. 4:e1000035. doi: 10.1371/ journal.ppat.1000035

Bai, Y. K., Zhou, K., and Doudna, J. A. (2013). Hepatitis C virus $3^{\prime}$ UTR regulates viral translation through direct interactions with the host translation machinery. Nucleic Acids Res. 41, 7861-7874. doi: 10.1093/nar/ gkt543

Bell, N. M., and Lever, A. M. L. (2013). HIV Gag polyprotein: processing and early viral particle assembly. Trends Microbiol. 21, 136-144. doi: 10.1016/j.tim.2012. 11.006

Bourlière, M., Gordon, S. C., Flamm, S. L., Cooper, C. L., Ramji, A., Tong, M., et al. (2017). Sofosbuvir, velpatasvir, and voxilaprevir for previously treated HCV infection. N. Engl. J. Med. 376, 2134-2146. doi: 10.1056/NEJMoa1613512 
Chamanian, M., Purzycka, K. J., Wille, P. T., Ha, J. S., McDonald, D., Gao, Y., et al. (2013). A cis-acting element in retroviral genomic RNA links GagPol ribosomal frameshifting to selective viral RNA encapsidation. Cell Host Microbe. 13, 181-192. doi: 10.1016/j.chom.2013.01.007

Chambers, T. J., Hahn, C. S., Galler, R., and Rice, C. M. (1990). Flavivirus genome organization, expression, and replication. Annu. Rev. Microbiol. 44, 649-688. doi: 10.1146/annurev.mi.44.100190.003245

Chen, J., Rahman, S. A., Nikolaitchik, O. A., Grunwald, D., Sardo, L., Burdick, R. C., et al. (2016). HIV-1 RNA genome dimerizes on the plasma membrane in the presence of Gag protein. Proc. Natl. Acad. Sci. U.S.A. 113, 201-208. doi: $10.1073 /$ pnas. 1518572113

Choo, Q. L., Kuo, G., Weiner, A. J., Overby, L. R., Bradley, D. W., and Houghton, M. (1989). Isolation of a cDNA clone derived from a blood-borne non-A, non-B viral hepatitis genome. Science 244, 359-362. doi: 10.1126/ science. 2523562

Clarke, B. D., Roby, J. A., Slonchak, A., and Khromykh, A. A. (2015). Functional non-coding RNAs derived from the flavivirus $3^{\prime}$ untranslated region. Virus Res. 206, 53-61. doi: 10.1016/j.virusres.2015.01.026

Comas-Garcia, M., Datta, S. A., Baker, L., Varma, R., Gudla, P. R., and Rein, A. (2017). Dissection of specific binding of HIV-1 Gag to the 'packaging signal' in viral RNA. Elife 6:e27055. doi: 10.7554/eLife.27055

Comas-Garcia, M., Davis, S. R., and Rein, A. (2016). On the selective packaging of genomic RNA by HIV-1. Viruses 8:E246. doi: 10.3390/v8090246

Cristofari, G., Ivanyi-Nagy, R., Gabus, C., Boulant, S., Lavergne, J. P., Penin, F., et al. (2004). The hepatitis $C$ virus core protein is a potent nucleic acid chaperone that directs dimerization of the viral (+) strand RNA in vitro. Nucleic Acids Res. 32, 2623-2631. doi: 10.1093/nar/gkh579

Diviney, S., Tuplin, A., Struthers, M., Armstrong, V., Elliott, R. M., Simmonds, P., et al. (2008). A hepatitis $C$ virus cis-acting replication element forms a longrange RNA-RNA interaction with upstream RNA sequences in NS5B. J. Virol. 82, 9008-9022. doi: 10.1128/JVI.02326-07

D'Souza, V., and Summers, M. F. (2005). How retroviruses select their genomes. Nat. Rev. Microbiol. 3, 643-655. doi: 10.1038/nrmicro1210

Fernández-Sanlés, A., Ríos-Marco, P., Romero-López, C., and Berzal-Herranz, A. (2017). Functional information stored in the conserved structural RNA domains of flavivirus genomes. Front. Microbiol. 8:546. doi: 10.3389/fmicb. 2017.00546

Freed, E. O. (2015). HIV-1 assembly, release and maturation. Nat. Rev. Microbiol. 13, 484-496. doi: 10.1038/nrmicro3490

Friebe, P., and Bartenschlager, R. (2002). Genetic analysis of sequences in the $3^{\prime}$ nontranslated region of hepatitis $\mathrm{C}$ virus that are important for RNA replication. J. Virol. 76, 5326-5338. doi: 10.1128/JVI.76.11.5326-5338.2002

Friebe, P., and Bartenschlager, R. (2009). Role of RNA structures in genome terminal sequences of the hepatitis $\mathrm{C}$ virus for replication and assembly. J. Virol. 83, 11989-11995. doi: 10.1128/JVI.01508-09

Friebe, P., Boudet, J., Simorre, J. P., and Bartenschlager, R. (2005). Kissingloop interaction in the $3^{\prime}$ end of the hepatitis $C$ virus genome essential for RNA replication. J. Virol. 79, 380-392. doi: 10.1128/JVI.79.1.380-392. 2005

Gawlik, K., and Gallay, P. A. (2014). HCV core protein and virus assembly: what we know without structures. Immunol. Res. 60, 1-10. doi: 10.1007/s12026-0148494-3

Guo, J., Wu, T., Kane, B. F., Johnson, D. G., Henderson, L. E., Gorelick, R. J., et al. (2002). Subtle alterations of the native zinc finger structures have dramatic effects on the nucleic acid chaperone activity of human immunodeficiency virus type 1 nucleocapsid protein. J. Virol. 76, 4370-4378. doi: 10.1128/JVI.76.9.43704378.2002

Harris, D., Zhang, Z., Chaubey, B., and Pandey, V. N. (2006). Identification of cellular factors associated with the $3^{\prime}$-nontranslated region of the hepatitis $\mathrm{C}$ virus genome. Mol. Cell Proteomics. 5, 1006-1018. doi: 10.1074/mcp.M500429MCP200

Honda, M., Brown, E. A., and Lemon, S. M. (1996). Stability of a stem-loop involving the initiator AUG controls the efficiency of internal initiation of translation on hepatitis C virus RNA. RNA. 2, 955-968.

Ivanyi-Nagy, R., Kanevsky, I., Gabus, C., Lavergne, J. P., Ficheux, D., Penin, F., et al. (2006). Analysis of hepatitis C virus RNA dimerization and coreRNA interactions. Nucleic Acids Res. 34, 2618-2633. doi: 10.1093/nar/ $\mathrm{gkl} 240$
Jopling, C. L. (2008). Regulation of hepatitis C virus by microRNA-122. Biochem. Soc. Trans. 36(Pt 6), 1220-1223. doi: 10.1042/BST0361220

Khromykh, A. A., Meka, H., Guyatt, K. J., and Westaway, E. G. (2001a). Essential role of cyclization sequences in flavivirus RNA replication. J. Virol. 75, 6719-6728. doi: 10.1128/JVI.75.14.6719-6728.2001

Khromykh, A. A., Varnavski, A. N., Sedlak, P. L., and Westaway, E. G. (2001b). Coupling between replication and packaging of flavivirus RNA: evidence derived from the use of DNA-based full-length cDNA clones of kunjin virus. J. Virol. 75, 4633-4640.

Lever, A., Gottlinger, H., Haseltine, W., and Sodroski, J. (1989). Identification of a sequence required for efficient packaging of human immunodeficiency virus type 1 RNA into virions. J. Virol. 63, 4085-4087.

Li, Q., Pène, V., Krishnamurthy, S., Cha, H., and Liang, T. J. (2013). Hepatitis $C$ virus infection activates an innate pathway involving IKK- $\alpha$ in lipogenesis and viral assembly. Nat. Med. 19, 722-729. doi: 10.1038/nm. 3190

Lu, K., Heng, X., Garyu, L., Monti, S., Garcia, E. L., Kharytonchyk, S., et al. (2011). NMR detection of structures in the HIV-1 5' -leader RNA that regulate genome packaging. Science 334, 242-245. doi: 10.1126/science.1210460

Machlin, E. S., Sarnow, P., and Sagan, S. M. (2011). Masking the $5^{\prime}$ terminal nucleotides of the hepatitis $\mathrm{C}$ virus genome by an unconventional microRNAtarget RNA complex. Proc. Natl. Acad. Sci. U.S.A. 108, 3193-3198. doi: 10.1073/ pnas. 1012464108

Masaki, T., Suzuki, R., Murakami, K., Aizaki, H., Ishii, K., Murayama, A., et al. (2008). Interaction of hepatitis $\mathrm{c}$ virus nonstructural protein $5 \mathrm{~A}$ with core protein is critical for the production of infectious virus particles. J. Virol. 82, 7964-7976. doi: 10.1128/JVI.00826-08

Masaki, T., Suzuki, R., Saeed, M., Mori, K., Matsuda, M., Aizaki, H., et al. (2010). Production of infectious hepatitis C virus by using RNA polymerase I-mediated transcription. J. Virol. 84, 5824-5835. doi: 10.1128/JVI.023 97-09

Masaki, T., and Suzuki, T. (2015). NS5A phosphorylation: its functional role in the life cycle of hepatitis C virus. Fut. Virol. 10, 751-762. doi: 10.1128/JVI.031 70-13

McBride, M. S., and Panganiban, A. T. (1996). The human immunodeficiency virus type 1 encapsidation site is a multipartite RNA element composed of functional hairpin structures. J. Virol. 70, 2963-2973.

Mir, M. A., Brown, B., Hjelle, B., Duran, W. A., and Panganiban, A. T. (2006). Hantavirus $\mathrm{N}$ protein exhibits genus-specific recognition of the viral RNA panhandle. J. Virol. 80, 11283-11292. doi: 10.1128/JVI.00 820-06

Moriishi, K., and Matsuura, Y. (2016). "Structural proteins of HCV and biological functions," in Hepatitis C Virus I, Cellular and Molecular Virology, eds T. Miyamura, S. M. Lemon, C. M. Walker, and T. Wakita (Berlin: Springer).

Nishikawa, F., Funaji, K., Fukuda, K., and Nishikawa, S. (2004). In vitro selection of RNA aptamers against the HCV NS3 helicase domain. Oligonucleotides 14, 114-129. doi: 10.1089/1545457041526335

Nugent, C. I., Johnson, K. L., Sarnow, P., and Kirkegaard, K. (1999). Functional coupling between replication and packaging of poliovirus replicon RNA. J. Virol. 73, 427-435.

Olson, E. D., Cantara, W. A., and Musier-Forsyth, K. (2015). New structure sheds light on selective HIV-1 genomic RNA packaging. Viruses 7, 4826-4835. doi: $10.3390 / \mathrm{v} 7082846$

Paillart, J. C., Shehu-Xhilaga, M., Marquet, R., and Mak, J. (2004). Dimerization of retroviral RNA genomes: an inseparable pair. Nat. Rev. Microbiol. 2, 461-472. doi: 10.1038/nrmicro903

Palau, W., Masante, C., Ventura, M., and Di, Primo C (2013). Direct evidence for RNA-RNA interactions at the $3^{\prime}$ end of the Hepatitis $C$ virus genome using surface plasmon resonance. RNA 19, 982-991. doi: 10.1261/rna.0376 06.112

Quadri, R., and Negro, F. (2001). Are there any subgenomic forms of hepatitis C virus RNA in the liver? Dig. Liver Dis. 33, 480-486. doi: 10.1016/S1590-8658(01) 80026-8

Romero-López, C., Barroso-Deljesus, A., García-Sacristán, A., Briones, C., and Berzal-Herranz, A. (2014). End-to-end crosstalk within the hepatitis C virus genome mediates the conformational switch of the $3^{\prime} \mathrm{X}$-tail region. Nucleic Acids Res. 42, 567-582. doi: 10.1093/nar/gkt841 
Romero-Lopez, C., and Berzal-Herranz, A. (2009). A long-range RNA-RNA interaction between the $5^{\prime}$ and $3^{\prime}$ ends of the HCV genome. RNA 15, 1740-1752. doi: 10.1261/rna.1680809

Russell, R. S., Hu, J., Bériault, V., Mouland, A. J., Laughrea, M., Kleiman, L., et al. (2003). Sequences downstream of the $5^{\prime}$ splice donor site are required for both packaging and dimerization of human immunodeficiency virus type 1 RNA. J. Virol. 77, 84-96. doi: 10.1128/JVI.77.1.84-96.2003

Shetty, S., Kim, S., Shimakami, T., Lemon, S. M., and Mihailescu, M. R. (2010). Hepatitis $\mathrm{C}$ virus genomic RNA dimerization is mediated via a kissing complex intermediate. RNA 16, 913-925. doi: 10.1261/rna.1960410

Shi, G., Ando, T., Suzuki, R., Matsuda, M., Nakashima, K., Ito, M., et al. (2016). Involvement of the $3^{\prime}$ untranslated region in encapsidation of the hepatitis $\mathrm{C}$ virus. PLoS Pathog. 12:e1005441. doi: 10.1371/journal.ppat.1005441

Shimizu, Y. K., Hijikata, M., Oshima, M., Shimizu, K., and Yoshikura, H. (2006). Detection of a $5^{\prime}$ end subgenome of hepatitis $C$ virus terminating at nucleotide 384 in patients' plasma and liver tissues. J. Viral. Hepat. 13, 746-755. doi: 10.1111/j.1365-2893.2006.00745.x

Skripkin, E., Paillart, J. C., Marquet, R., Ehresmann, B., and Ehresmann, C. (1994). Identification of the primary site of the human immunodeficiency-virus type1 RNA dimerization in-vitro. Proc. Natl. Acad. Sci. U.S.A. 91, 4945-4949. doi: 10.1073/pnas.91.11.4945

Song, Y., Friebe, P., Tzima, E., Jünemann, C., Bartenschlager, R., and Niepmann, M. (2006). The hepatitis C virus RNA $3^{\prime}$-untranslated region strongly enhances translation directed by the internal ribosome entry site. J. Virol. 80, 11579-11588. doi: 10.1128/JVI.00675-06

Stewart, H., Bingham, R. J., White, S. J., Dykeman, E. C., Zothner, C., Tuplin, A. K., et al. (2016). Identification of novel RNA secondary structures within the hepatitis $\mathrm{C}$ virus genome reveals a cooperative involvement in genome packaging. Sci. Rep. 6:22952. doi: 10.1038/srep22952

Suzuki, T. (2017). Hepatitis C virus replication. Adv. Exp. Med. Biol. 997, 199-209. doi: 10.1007/978-981-10-4567-7_15

Telesnitsky, A., and Wolin, S. L. (2016). The host RNAs in retroviral particles. Viruses 8:E235. doi: 10.3390/v8080235
Tuplin, A., Struthers, M., Simmonds, P., and Evans, D. J. (2012). A twist in the tail: shape mapping of long-range interactions and structural rearrangements of RNA elements involved in HCV replication. Nucleic Acids Res. 40, 6908-6921. doi: 10.1093/nar/gks370

Villordo, S. M., and Gamarnik, A. V. (2009). Genome cyclization as strategy for flavivirus RNA replication. Virus Res. 139, 230-239. doi: 10.1016/j.virusres. 2008.07.016

Wang, C., Le, S. Y., Ali, N., and Siddiqui, A. (1995). An RNA pseudoknot is an essential structural element of the internal ribosome entry site located within the hepatitis $\mathrm{C}$ virus $5^{\prime}$ noncoding region. RNA 1 , 526-537.

Webb, J. A., Jones, C. P., Parent, L. J., Rouzina, I., and Musier-Forsyth, K. (2013). Distinct binding interactions of HIV-1 Gag to Psi and non-Psi RNAs: implications for viral genomic RNA packaging. RNA 19, 1078-1088. doi: 10. $1261 /$ rna.038869.113

You, S., and Rice, C. M. (2008). $3^{\prime}$ RNA elements in hepatitis C virus replication: kissing partners and long poly(U). J. Virol. 82, 184-195. doi: 10.1128/JVI.017 96-07

You, S., Stump, D. D., Branch, A. D., and Rice, C. M. (2004). A cis-acting replication element in the sequence encoding the NS5B RNA-dependent RNA polymerase is required for hepatitis C virus RNA replication. J. Virol. 78, 1352-1366. doi: 10.1128/JVI.78.3.1352-1366.2004

Conflict of Interest Statement: The authors declare that the research was conducted in the absence of any commercial or financial relationships that could be construed as a potential conflict of interest.

Copyright (c) 2018 Shi and Suzuki. This is an open-access article distributed under the terms of the Creative Commons Attribution License (CC BY). The use, distribution or reproduction in other forums is permitted, provided the original author(s) and the copyright owner are credited and that the original publication in this journal is cited, in accordance with accepted academic practice. No use, distribution or reproduction is permitted which does not comply with these terms. 